\title{
Luminescence of Highly Excited Nonpolar a-Plane GaN and AlGaN/GaN Multiple Quantum Wells
}

S. JuršĖnas*, E. KuOKŠTis, S. Miasojedovas, G. KurilČIK, A. Z̆UKAuskas

Institute of Materials Science and Applied Research, Vilnius University Sauletekio 9-III, 10222 Vilnius, Lithuania

C.Q. Chen, J.W. Yang, V. Adivarahan and M. Asif Khan Department of Electrical Engineering, University of South Carolina Columbia, SC 29208, USA

\begin{abstract}
Carrier recombination dynamics in polar and nonpolar GaN epilayers and GaN/AlGaN multiple quantum wells grown over sapphire substrates with various crystallographic orientation were studied under high photoexcitation by 20 ps laser pulses. The transient of luminescence featured a significant enhancement in nonradiative recombination of free carriers for nonpolar a-plane GaN epilayers compared to conventional $c$-plane samples. The epitaxial lateral overgrowth technique was demonstrated to significantly improve the quality of nonpolar $a$-plane films. This was proved by more than 40 -fold increase in luminescence decay time ( $430 \mathrm{ps}$ compared to $\leq 10 \mathrm{ps}$ in the ordinary $a$-plane epilayer). Under high-excitation regime, a complete screening of built-in electric field by free carriers in multiple quantum wells grown on $c$-plane and $r$-plane sapphire substrates was achieved. Under such high excitation, luminescence efficiency and carrier lifetime of multiple quantum wells was shown to be determined by the substrate quality.
\end{abstract}

PACS numbers: $78.55 . \mathrm{Cr}, 73.21 . \mathrm{Fg}, 72.20 . \mathrm{Jv}, 78.47 .+\mathrm{p}$

\section{Introduction}

Group-III nitride materials are widely used for fabrication of UV-blue light emitting diodes and lasers [1]. Recent progress in growth of GaN epilayers with

${ }^{*}$ corresponding author; e-mail: saulius.jursenas@ff.vu.lt 
nonpolar orientation and corresponding nitride-based heterostructures opens new prospective in design and application of electronic and optoelectronic devices without the restrictions imposed by strong built-in electric fields, which are always present in conventional $c$-plane ([0001]-oriented) group-III nitride heterostructures [2-9]. One of the most powerful tools used for materials characterization is luminescence spectroscopy. However, optical properties under high excitation conditions, which are typical of operating regimes of laser and high-power light-emitting diodes, have not been investigated in $a$-plane oriented films so far. Here, we report on luminescence properties of group-III nitrides and their multiple quantum wells (MQWs) with polar and nonpolar orientation revealed under strong laser excitation.

\section{Experimental}

The $c$-plane GaN epilayers were grown on $c$-plane sapphire substrates using a low-pressure metalorganic chemical vapor deposition (MOCVD) reactor. We used triethylgallium and $\mathrm{NH}_{3}$ as the precursors for Ga and $\mathrm{N}$, respectively. A $1.5 \mu \mathrm{m}$ thick GaN epilayer was deposited on the sapphire substrate with a $20 \mathrm{~nm}$ thick low-temperature GaN buffer layer. Hydrogen was used as a carrier gas and the reactor pressure was kept at 76 Torr. The a-plane GaN epilayer was grown over the $r$-plane sapphire substrates using a similar two-step growth procedure $[8,9]$. The growth was carried out at $1080^{\circ} \mathrm{C}$ and at a pressure of 50 Torr. The thickness of GaN epilayer was around $1.5 \mu \mathrm{m}$. Subsequently, a $0.2 \mu \mathrm{m}$ thick $\mathrm{SiO}_{2}$ was deposited by plasma enhanced chemical vapor deposition on a part of the a-plane GaN epilayers for fully coalesced epitaxial lateral overgrowth (ELOG). The mask pattern consisted of $12 \mu \mathrm{m}$ wide $\mathrm{SiO}_{2}$ stripes and $6 \mu \mathrm{m}$ wide opening windows that were fabricated by using standard photolithography and wet etching. The stripes were oriented along the [1100] direction. The samples were then reloaded in the MOCVD system and additional growth was carried out at $1080^{\circ} \mathrm{C}$ for two hours and $1050^{\circ} \mathrm{C}$ for three hours. The lower temperature caused an enhanced lateral growth rate that resulted in a full coalescence of the GaN stripes. Under our growth conditions, the lateral to vertical growth rate ratio was around 3:5. Additionally, three MQW samples were prepared. $5 \mathrm{MQWs}$, consisting of $5 \mathrm{~nm}$ GaN quantum wells and $8 \mathrm{~nm} \mathrm{AlGaN} \mathrm{barriers} \mathrm{(approximately} \mathrm{25 \%} \mathrm{Al)} \mathrm{pairs} \mathrm{were}$ grown on GaN epilayer that was deposited over $c$ - and $r$-planes of the sapphire substrates.

The samples were excited by the fourth harmonic (photon energy $h \nu_{\mathrm{g}}=$ $4.66 \mathrm{eV}$ ) of the actively-passively mode-locked YAG: $\mathrm{Nd}^{3+}$ laser (pulse duration was $20 \mathrm{ps}$, repetition rate was $2.7 \mathrm{~Hz}$, and maximum pump energy was $40 \mu \mathrm{J}$ ). The size of the excitation spot was approximately $1 \mathrm{~mm}$. Photoluminescence was collected in backscattering geometry and dispersed by a $0.4 \mathrm{~m}$ grating monochromator. Toluene optical Kerr shutter was used for temporal resolution (20 ps) of the luminescence decay. The experiments were carried out at room temperature. 


\section{Results and discussion}

Figure 1 displays typical time-integrated photoluminescence (PL) spectra for $a$-plane and $c$-plane GaN, as well as for $a$-plane ELOG GaN for the excitation energy density of $I_{\mathrm{g}}=0.3 \mathrm{~mJ} / \mathrm{cm}^{2}$. At high photoexcitation, the spectra of $a$-plane samples consist of one broad emission band peaked in the vicinity of the band-gap energy $(\approx 3.4 \mathrm{eV})$. The observed luminescence spectra are typical of the radiative recombination of high-density electron-hole plasma (EHP) that occurs under intense photoexcitation with the excitonic states screened by the nonequilibrium carrier system [10-12]. The EHP band dominates in the emission in a broad range of excitation intensities. For the highest excitation $I_{\mathrm{g}}>3 \mathrm{~mJ} / \mathrm{cm}^{2}$,

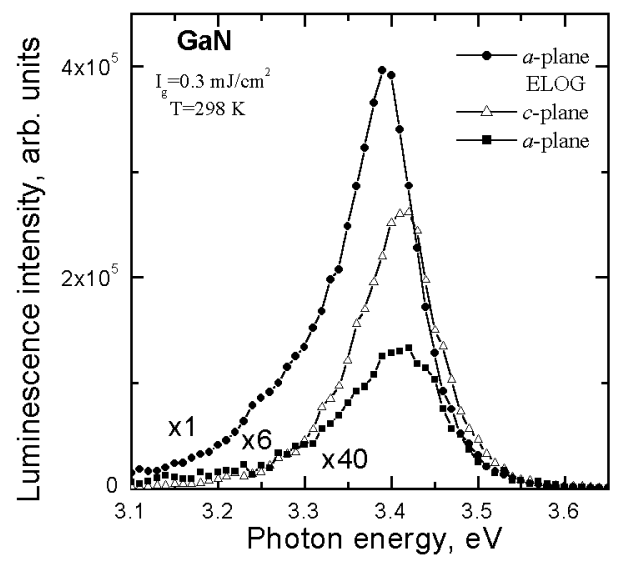

Fig. 1. Time-integrated luminescence spectra of the $c$-plane (triangles), a-plane (squares), and a-plane ELOG (circles) GaN films for the excitation energy density $I_{\mathrm{g}}=0.3 \mathrm{~mJ} / \mathrm{cm}^{2}$.

the spectra are modified due to stimulated emission and carrier heating effects. PL intensity is seen to differ significantly in different samples. Namely, the $a$-plane GaN epilayers grown over $r$-plane sapphire exhibit a decrease in EHP luminescence intensity by about 20 times in comparison with the conventional $c$-plane GaN epilayers grown over $c$-plane sapphire, whereas the $a$-plane ELOG structure demonstrates 110 times higher emission intensity than that of the $a$-plane $\mathrm{GaN}$ epilayer. Accordingly, the peak position of spontaneous emission is at $3.42 \mathrm{eV}$, $3.42 \mathrm{eV}$, and $3.39 \mathrm{eV}$ for the $a$-plane epilayer, $c$-plane epilayer, and $a$-plane ELOG film, respectively. The increase in the emission intensity in the ELOG film can be accounted for by an increase in EHP lifetime and, consequently, the redshift can be attributed to band-gap renormalization due to generation of plasma with higher initial density.

Figure 2 shows the emission intensity as a function of time at the peak position of the PL band for the excitation intensity $I_{\mathrm{g}}=0.8 \mathrm{~mJ} / \mathrm{cm}^{2}$ in three 


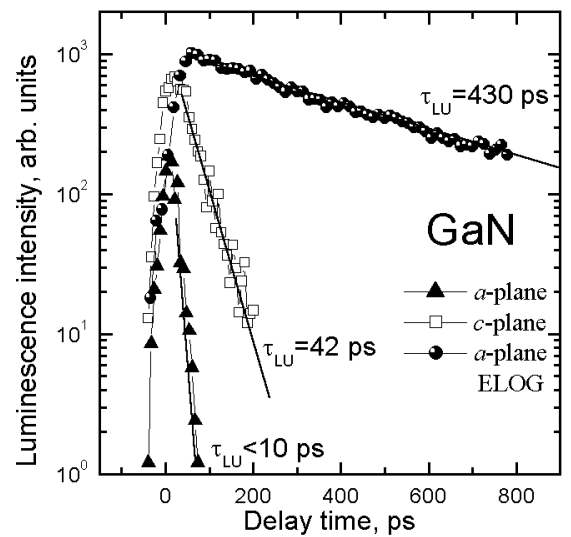

Fig. 2. Transient behavior of the luminescence intensity at the peak position of spontaneous emission in the a-plane (triangles), c-plane (squares), and the $a$-plane ELOG (circles) GaN films for the excitation energy density $I_{\mathrm{g}}=0.8 \mathrm{~mJ} / \mathrm{cm}^{2}$.

types of epilayers. As one can see, the luminescence decay is nearly exponential with a characteristic time constant of $\tau_{\mathrm{LU}} \leq 10 \mathrm{ps}, \tau_{\mathrm{LU}}=42 \mathrm{ps}$, and $\tau_{\mathrm{LU}}=430 \mathrm{ps}$ for the $a$-plane, $c$-plane, and $a$-plane ELOG GaN epilayers, respectively. The first two values were estimated by a deconvolution procedure. The obtained variation in luminescence decay times is in good agreement with the experimentally observed time-integrated luminescence intensity (Fig. 1).

The observed dynamics of the PL spectra can be understood as follows. Usually, structures grown over $c$-plane sapphire substrates have smoother surfaces and higher quality alloys as compared with films grown over $r$-plane sapphire substrates [13]. Thus, the rate of nonradiative recombination of EHP is higher in the a-plane GaN epilayer grown over $r$-plane sapphire in comparison with the conventional c-plane epilayer. Our results show that the ELOG procedure significantly improves the $a$-plane GaN material quality and reduces the influence of the substrate-related defects. This results in more than a 40-fold increase in the value of the luminescence decay time $\left(\tau_{\mathrm{LU}}=430 \mathrm{ps}\right)$ and the PL intensity for the ELOG sample in comparison with the ordinary $a$-plane sample $(\leq 10 \mathrm{ps})$. The exponential decay profile of the luminescence intensity implies the deep-trap saturation regime, which can be easily achieved in GaN epilayers under high excitation conditions $\left(\approx 1 \mathrm{~mJ} / \mathrm{cm}^{2}\right)[11,12]$. Under such a regime, the characteristic PL decay time is related to the time $\tau_{\mathrm{e}}$ of free-carrier capture to the deep traps as $\tau_{\mathrm{LU}} \cong \tau_{\mathrm{e}} / 2$. For the nonpolar $a$-plane ELOG film, the estimated carrier lifetime at room temperature is $\tau_{\mathrm{e}} \cong 860 \mathrm{ps}$ that is close to the value $\tau_{\mathrm{e}} \cong 970 \mathrm{ps}$ observed in almost dislocation-free homoepitaxial GaN epilayer [11].

Figure 3 shows typical time-integrated PL spectra of AlGaN/GaN MQWs grown on the $a$-plane and the $c$-plane $\mathrm{GaN}$, for the excitation energy density of $I_{\mathrm{g}}=0.3 \mathrm{~mJ} / \mathrm{cm}^{2}$. Due to spatial confinement effect in the GaN quantum wells, the 


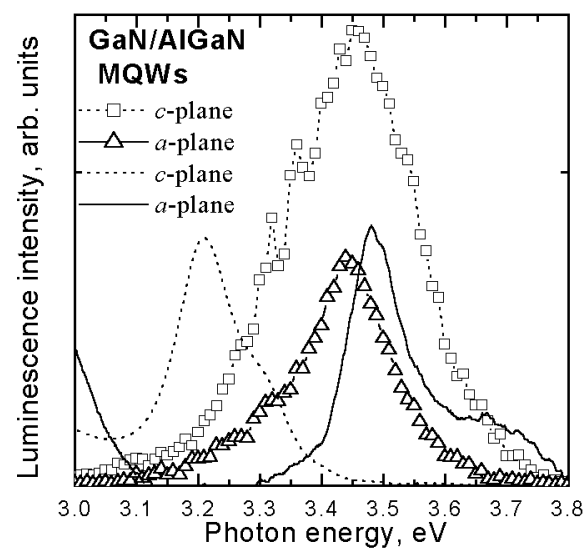

Fig. 3. Time-integrated luminescence spectra of the $a$-plane (triangles) and c-plane (squares) AlGaN/GaN MQWs for the excitation energy density $I_{\mathrm{g}}=0.3 \mathrm{~mJ} / \mathrm{cm}^{2}$. The normalized luminescence spectra for low $I_{\mathrm{g}}=16 \mu \mathrm{J} / \mathrm{cm}^{2}$ excitation are shown for comparison: dotted line - c-plane MQW, solid line $-c$-plane MQW.

spectra are slightly blueshifted compared to corresponding ones of GaN epilayer emission (Fig. 1). The low-energy edge of the c-plane MQWs emission is obviously distorted by stimulated transitions. The PL peak position in both the polar and nonpolar MQWs is close to $3.45 \mathrm{eV}$ indicating on screening of built-in field by free carriers (the nonequilibrium-carrier density is of the order of $10^{19} \mathrm{~cm}^{-3}$ ). Luminescence of the $c$-plane MQW is twice more intense, compared to a-plane MQW. For comparison, lines in Fig. 3 show normalized luminescence spectra of the same structures obtained at low $16 \mu \mathrm{J} / \mathrm{cm}^{2}$ excitation (excimer laser, $8 \mathrm{~ns}$ duration, $6.42 \mathrm{eV}$ photon energy pulses). Luminescence of the nonpolar $a$-plane MQWs is po-

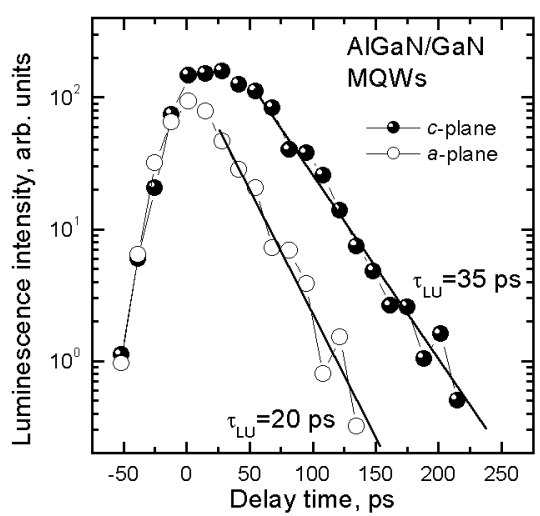

Fig. 4. Transient behavior of the luminescence intensity at the peak position of spontaneous emission in the a-plane (open circles) and $c$-plane (solid circles) AlGaN/GaN MQWs for the excitation energy density $I_{\mathrm{g}}=0.8 \mathrm{~mJ} / \mathrm{cm}^{2}$. 
sitioned at $3.481 \mathrm{eV}$, i.e. it is slightly blueshifted compared to high excitation, while the $c$-plane grown MQWs exhibit significantly redshifted luminescence at $3.21 \mathrm{eV}$ due to in-well electric field. Since at high excitation polarization field is screened by nonequilibrium carriers, the luminescence lifetime reflects carrier capture rate, which is solely characteristics of the material. Thus the materials quality can be compared in MQWs grown on substrates of different orientation. Figure 4 shows luminescence transients obtained at the peak position for the $c$-plane and $a$-plane MQWs. The luminescence decay time in polar $c$-plane MQW $\left(\tau_{\mathrm{LU}}=35 \mathrm{ps}\right)$ is larger than that in $a$-plane structure $\left(\tau_{\mathrm{LU}}=20 \mathrm{ps}\right)$ which correlates with the total intensity of the corresponding emission bands. Thus, although a MQW structure slightly suppresses the impact of the threading dislocations, the materials quality of the $a$-plane MQWs grown over $r$-plane sapphire is lower than that of the MQWs grown over $c$-plane sapphire.

\section{Conclusions}

In conclusion, transient luminescence dynamics under high excitation conditions was studied in GaN epilayers and MQWs of polar (c-plane) and nonpolar (a-plane) orientation. The model of dense electron-hole plasma recombination was applied for characterization of materials quality. Luminescence transients were explained in terms of free carrier capture to the saturated deep centers of nonradiative recombination. Nonpolar $a$-plane epilayers were shown to possess a large amount of nonradiative traps, which significantly diminishes both the carrier lifetime and luminescence efficiency. Application of epitaxial lateral overgrowth technique was shown to be capable of significant reduction of nonradiative capture in $a$-plane GaN films.

Under high-excitation regime, complete screening of built-in electric field by free carriers was realized in AlGaN/GaN MQWs grown on $c$-plane and $r$-plane sapphire substrates. This enabled materials characterization by the carrier lifetime. In highly photoexcited MQWs, the luminescence efficiency and carrier lifetime was shown to be determined by the substrate quality.

\section{Acknowledgments}

The research at Vilnius University was partially supported by the Lithuanian State Science and Education Foundation under COST529 program and European Commission supported SELITEC center contract No. G5MA-CT-2002-04047. A.Z̆. acknowledges the Lithuanian Ministry of Education and Science for his fellowship. 


\section{References}

[1] S. Nakamura, G. Fasol, The Blue Laser Diode: GaN Based Light Emitters and Lasers, Springer, Berlin 1997.

[2] P. Waltereit, O. Brandt, M. Ramsteiner, R. Uecker, P. Reiche, K.H. Ploog, J. Cryst. Growth 218, 143 (2000).

[3] M.D. Craven, S.H. Lim, F. Wu, J.S. Speck, S.P. DenBaars, Appl. Phys. Lett. 81, 1201 (2002).

[4] H.M. Ng, Appl. Phys. Lett. 80, 4369 (2002).

[5] M.D. Craven, P. Waltereit, J.S. Speck, S.P. DenBaars, Appl. Phys. Lett. 84, 496 (2004).

[6] E. Kuokstis, C.Q. Chen, M.E. Gaevski, W.H. Sun, J.W. Yang, G. Simin, M. Asif Khan, H.P. Maruska, D.W. Hill, M.C. Chou, J.J. Gallagher, B. Chai, Appl. Phys. Lett. 81, 4130 (2002).

[7] Y.J. Sun, O. Brandt, S. Cronenberg, S. Dhar, H.T. Grahn, K.H. Ploog, P. Waltereit, J.S. Speck, Phys. Rev. B 67, 041306 (2003).

[8] W.H.Sun, J. W. Yang, C.Q. Chen, J.P. Zhang, M.E. Gaevski, E. Kuokstis, V. Adivarahan, H.M. Wang, Z. Gong, M. Su, M.A. Khan, Appl. Phys. Lett. 83, 2599 (2003).

[9] C.Q. Chen, J.W. Yang, H.M. Wang, J.P. Zhang, V. Adivarahan, M. Gaevski, E. Kuokstis, Z. Gong, M. Su, M.A. Khan, Jpn. J. Appl. Phys. 42, L640 (2003).

[10] F. Binet, J.Y. Duboz, J. Off, F. Scholz, Phys. Rev. B 60, 4715 (2099).

[11] S. Juršenas, G. Kurilčik, N. Kurilčik, A. Žukauskas, P. Prystawko, M. Leszczynski, T. Suski, P. Perlin, I. Grzegory, S. Porowski, Appl. Phys. Lett. 78, 3776 (2001).

[12] S. Juršenas, S. Miasojedovas, G. Kurilčik, A. Žukauskas, P.R. Hageman, Appl. Phys. Lett. 83, 66 (2003).

[13] W.A. Melton, J.I. Pankove, J. Cryst. Growth 178, 168 (1997). 